KYUNGPOOK Math. J. 50(2010), 71-80

\title{
Unicity of Meromorphic Function and its Derivative
}

\author{
Ang Chen* AND Guowei Zhang \\ Department of Mathematics, Shandong University, Jinan 250100, P. R. Chian \\ e-mail : ang.chen.jr@gmail.com and zhirobo@yahoo.com.cn
}

ABstract. In this paper, we deal with the uniqueness problems of meromorphic functions that share a small function with its derivative and improve some results of Yang, Yu, Lahiri, and Zhang, also answer some questions of T. D. Zhang and W. R. Lü.

\section{Introduction and main results}

In this article, by meromorphic functions we shall always mean meromorphic functions in the complex plane. we are going to mainly use the basic notation of Nevanlinna Theory, (see [1], [3], [2]) such as $T(r, f), N(r, f), m(r, f), \bar{N}(r, f)$ and $S(r, f)=o(T(r, f))$. Let $f(z)$ and $g(z)$ denote two non-constant meromorphic functions, and let $a(z)$ be a meromorphic function. If $f(z)-a(z)$ and $g(z)-a(z)$ have the same zeros with the same multiplicities(ignoring multiplicities), then we say that $f(z)$ and $g(z)$ share $a(z) \mathrm{CM}(\mathrm{IM})$. Let $k$ be a positive integer. We denote by $N_{k)}(r, 1 / f-a)$ the counting function for the zeros of $f-a$ with multiplicity $\leq k$, and by $\bar{N}_{k)}(r, 1 / f-a)$ the corresponding one for which the multiplicity is not counted. Let $N_{(k}(r, 1 / f-a)$ be the counting function for the zeros of $f-a$ with multiplicity $\geq k$, and $\bar{N}_{(k}(r, 1 / f-a)$ be the corresponding one for which the multiplicity is not counted. Set $N_{k}(r, 1 / f-a)=\bar{N}(r, 1 / f-a)+\bar{N}_{(2}(r, 1 / f-a)+\cdots+\bar{N}_{(k}(r, 1 / f-a)$. And we define

$$
\delta_{p}(a, f)=1-\limsup _{r \rightarrow+\infty} \frac{N_{p}(r, 1 / f-a)}{T(r, f)} .
$$

Obviously, $1 \geq \Theta(a, f) \geq \delta_{p}(a, f) \geq \delta(a, f) \geq 0$.

In 1996, Brück(see [6]) posed the following conjecture.

Conjecture. Let $f$ be a non-constant entire function such that the hyper-order $\sigma_{2}(f)$ of $f$ is not a positive integer and $\sigma_{2}(f)<\infty$. If $f$ and $f^{\prime}$ share a finite value $a \mathrm{CM}$, then $\frac{f^{\prime}-a}{f-a}=c$, where $\mathrm{c}$ is nonzero constant.

\footnotetext{
* Corresponding author.

Received April 30, 2009; revised August 9, 2009; accepted August 20, 2009.

2000 Mathematics Subject Classification: 30D35.

Key words and phrases: Meromorphic functions, small functions, value sharing, uniqueness.

The authors were supported by the NNFC of China (No.10771121), the NSF of Shang-dong Province, China (No Z2008A01).
} 
In [6], Brück proved under additional hypothesis that the conjecture holds when $a=1$, in fact he proved:

Theorem A. Let $f$ be non-constant entire funciton. If $f$ and $f^{\prime}$ share the value $1 C M$ and if $N\left(r, 1 / f^{\prime}\right)=S(r, f)$, then $\left(f^{\prime}-1\right) \backslash(f-1) \equiv c$ for some constant $c \in \mathbb{C} \backslash\{0\}$.

After that many people extended Theorem A and obtained many excellent results, such as L. Z. Yang[7], Q. C. Zhang[8] and Yu[9].

Theorem $\mathbf{B}([8])$. Let $f$ be a non-constant meromorphic function and $k$ be a positive integer. Suppose that $f$ and $f^{(k)}$ share $1 C M$ and

$$
2 \bar{N}(r, f)+\bar{N}\left(r, 1 / f^{\prime}\right)+N\left(r, 1 / f^{(k)}\right)<(\lambda+o(1)) T\left(r, f^{(k)}\right),
$$

for $r \in I$, where $I$ is a set of infinite linear measure and $\lambda$ satisfies $0<\lambda<1$, then $\left(f^{(k)}-1\right) \backslash(f-1) \equiv c$ for some constant $c \in \mathbb{C} \backslash\{0\}$.

Theorem $\mathbf{C}([\mathbf{1}])$. Let $f$ be a non-constant meromorphic function and $a(z)(\not \equiv 0, \infty)$ be a small function with respect to $f$, If

(1) $f$ and $g$ have no common poles,

(2) $f-a$ and $f^{(k)}-a$ share the value $0 C M$,

(3) $4 \delta(0, f)+2(k+8) \Theta(\infty, f)>2 k+19$,

then $f \equiv f^{(k)}$, where $k$ is a positive integer.

T.D.Zhang and W.R.Lü[10] recently considered the problem of a meromorphic function sharing one small function with its $\mathrm{k}$-th derivative and proved the following two theorems.

Theorem D. Let $k(\geq 1), n(\geq 1)$ be integers and $f$ be a non-constant meromorphic function. Also let $a \equiv a(z)(\not \equiv 0, \infty)$ be a meromorphic functions such that $T(r, a)=$ $S(r, f)$, as $r \rightarrow \infty$. Suppose that $f^{n}$ and $f^{(k)}$ share $a(z)$ IM and

$$
4 \bar{N}(r, f)+2 \bar{N}\left(r, \frac{1}{\left(f^{n} / a\right)^{\prime}}\right)+2 N_{2}\left(r, \frac{1}{f^{(k)}}\right)+\bar{N}\left(r, \frac{1}{f^{(k)}}\right)<(\lambda+o(1)) T\left(r, f^{(k)}\right),
$$

or $f^{n}$ and $f^{(k)}$ share $a(z) C M$ and

$$
2 \bar{N}(r, f)+\bar{N}\left(r, \frac{1}{\left(f^{n} / a\right)^{\prime}}\right)+N_{2}\left(r, \frac{1}{f^{(k)}}\right)<(\lambda+o(1)) T\left(r, f^{(k)}\right),
$$

for $0<\lambda<1, r \in I$, here $I$ is a set of infinite linear measure, then $\frac{f^{(k)}-a}{f^{n}-a} \equiv c$ for some constant $c \in \mathbb{C} \backslash\{0\}$.

Theorem E. Let $k(\geq 1), n(\geq 1)$ be integers and $f$ be a non-constant meromorphic function. Also let $a(z)(\equiv \equiv, \infty)$ be a small function with respect to $f$. If $f^{n}$ and $f^{(k)}$ share $a(z)$ IM and

$$
(2 k+6) \Theta(\infty, f)+4 \Theta(0, f)+2 \delta_{k+2}(0, f)>2 k+12-n,
$$


or $f^{n}$ and $f^{(k)}$ share $a(z) C M$ and

$$
(k+3) \Theta(\infty, f)+2 \Theta(0, f)+\delta_{k+2}(0, f)>k+6-n,
$$

then $f^{n} \equiv f^{(k)}$.

At the end of [10] T.D.Zhang and W.R.Lü asked a question: What will happen if $f^{n}$ and $\left[f^{(k)}\right]^{m}$ share a small function? We consider the problem and get our theorems as follow.

Theorem 1. Let $k(\geq 1), n(\geq 1), m(\geq 1)$ be integers and $f$ be a non-constant meromorphic function. Also let $a(z)(\not \equiv 0, \infty)$ be a small function with respect to $f$. If $f^{n}$ and $\left[f^{(k)}\right]^{m}$ share $a(z) I M$, and

$$
\begin{aligned}
& \frac{1}{m}\left[4 \bar{N}(r, f)+2 \bar{N}\left(r, \frac{1}{\left(f^{n} / a\right)^{\prime}}\right)+2 N_{2}\left(r, \frac{1}{f^{(k)}}\right)+\bar{N}\left(r, \frac{1}{f^{(k)}}\right)\right] \\
& <(\lambda+o(1)) T\left(r, f^{(k)}\right),
\end{aligned}
$$

or $f^{n}$ and $\left[f^{(k)}\right]^{m}$ share $a(z) C M$, and

$$
\frac{1}{m}\left[2 \bar{N}(r, f)+\bar{N}\left(r, \frac{1}{\left(f^{n} / a\right)^{\prime}}\right)+N_{2}\left(r, \frac{1}{f^{(k)}}\right]<(\lambda+o(1)) T\left(r, f^{(k)}\right),\right.
$$

for $0<\lambda<1, r \in I$, and $I$ is a set of infinite linear measure. Then $\left(\left[f^{(k)}\right]^{m}-a\right) \backslash$ $\left(f^{n}-a\right) \equiv c$ for some constant $c \in \mathbb{C} \backslash\{0\}$.

Theorem 2. Let $k(\geq 1), n(\geq 1), m(\geq 1)$ be integers and $f$ be a non-constant meromorphic function. Also let $a(z)(\not \equiv 0, \infty)$ be a small function with respect to $f$. If $f^{n}$ and $\left[f^{(k)}\right]^{m}$ share $a(z)$ IM and

$$
(2 k+6) \Theta(\infty, f)+3 \Theta(0, f)+2 \delta_{k+2}(0, f)>2 k+11-n,
$$

or $f^{n}$ and $\left[f^{(k)}\right]^{m}$ share $a(z) C M$ and

$$
(k+3) \Theta(\infty, f)+\delta_{2}(0, f)+2 \delta_{k+2}(0, f)>k+5-n,
$$

then $f^{n} \equiv\left[f^{(k)}\right]^{m}$.

Though we use the standard notations and definitions of the value distribution theory, we will explain some definitions and notations which are used in the paper.

Definition 1.1. Let $F$ and $G$ be two meromorphic functions defined in $\mathbb{C}$, assume $F$ and $G$ share $1 \mathrm{IM}$, let $z_{0}$ be a zero of $F-1$ with multiplicity $p$ and a zero of $G-1$ with multiplicity $q$. We denote by $N_{E}^{1)}(r, 1 / F-1)$ the counting function of the zeros of $F-1$ where $p=q=1$; by $N_{E}^{(2}(r, 1 / F-1)$ the counting funciton of zeros of $F-1$ where $p=q \geq 2$. We denote by $N_{L}(r, 1 / F-1)$ the counting 
function of the zeros of $F-1$ where $p>q \geq 1$; each point is counted according to its multiplicity, and $\bar{N}_{L}(r, 1 / F-1)$ denote its reduced form. In the same way, we can define $N_{E}^{1)}(r, 1 / G-1), N_{E}^{(2}(r, 1 / G-1), \bar{N}_{L}(r, 1 / G-1)$ and so on.

Definition 1.2. In this paper $N_{0}\left(r, \frac{1}{F^{\prime}}\right)$ denotes the counting function of the zeros of $F^{\prime}$ which are not the zeros of $F$ and $F-1$, and $\bar{N}_{0}\left(r, \frac{1}{F^{\prime}}\right)$ denote its reduced form. In the same way, we can define $N_{0}\left(r, \frac{1}{G^{\prime}}\right)$ and $\bar{N}_{0}\left(r, \frac{1}{G^{\prime}}\right)$.

\section{Some lemmas}

In this section we present some lemmas which will be needed in the sequel. Let $F, G$ be two non-constant meromorphic functions defined in $\mathbb{C}$. We shall denote by $H$ the following function.

$$
H=\left(\frac{F^{\prime \prime}}{F^{\prime}}-2 \frac{F^{\prime}}{F-1}\right)-\left(\frac{G^{\prime \prime}}{G^{\prime}}-2 \frac{G^{\prime}}{G-1}\right) .
$$

Lemma 2.1([1]). Let $f$ be a meromorphic function and $a$ is a finite complex number. Then

(i) $T\left(r, \frac{1}{f-a}\right)=T(r, f)+O(1)$,

(ii) $m\left(r, \frac{f^{(k)}}{f^{(l)}}\right)=S(r, f)$ for $k>l \geq 0$,

(iii) $T(r, f) \leq \bar{N}(r, f)+\bar{N}\left(r, \frac{1}{f-a_{1}(z)}\right)+\bar{N}\left(r, \frac{1}{f-a_{2}(z)}\right)+S(r, f)$,

where $a_{1}(z), a_{2}(z)$ are two meromorphic functions such that $T\left(r, a_{i}\right)=S(r, f)$, $(i=1,2)$.

Lemma 2.2(see page 354 in [3]). Let $F, G$ be two nonconstant meromorphic functions defined in $\mathbb{C}$. If $H \not \equiv, F$ and $G$ sharing 1 IM. Then

$$
\begin{aligned}
N(r, H) \leq & \bar{N}(r, F)+\bar{N}_{(2}\left(r, \frac{1}{F}\right)+\bar{N}_{(2}\left(r, \frac{1}{G}\right)+\bar{N}_{L}\left(r, \frac{1}{F-1}\right) \\
& +\bar{N}_{L}\left(r, \frac{1}{G-1}\right)+\bar{N}_{0}\left(r, \frac{1}{F^{\prime}}\right)+\bar{N}_{0}\left(r, \frac{1}{G^{\prime}}\right)+S(r, f) .
\end{aligned}
$$

If $F$ and $G$ sharing $1 C M$. Then

$$
N(r, H) \leq \bar{N}(r, F)+\bar{N}_{(2}\left(r, \frac{1}{F}\right)+\bar{N}_{(2}\left(r, \frac{1}{G}\right)+\bar{N}_{0}\left(r, \frac{1}{F^{\prime}}\right)+\bar{N}_{0}\left(r, \frac{1}{G^{\prime}}\right)+S(r, f) .
$$

Lemma 2.3([11]). Let $f$ be a non-constant meromorphic function and $n$ be a 
positive integer. $P(f)=a_{n} f^{n}+a_{n-1} f^{n-1}+\cdots+a_{1} f$, where $a_{i}$ are meromorphic functions such that $T\left(r, a_{i}\right)=S(r, f)(i=1,2, \ldots, n)$ and $a_{n} \not \equiv 0$. Then

$$
T(r . P(f))=n T(r, f)+S(r, f) .
$$

Lemma 2.4([5]). Let $f$ be a non-constant meromorphic function, $k$ be a positive integer. Then

$$
N_{p}\left(r, \frac{1}{f^{(k)}}\right) \leq N_{(p+k)}\left(r, \frac{1}{f}\right)+k \bar{N}(r, f)+S(r, f) .
$$

\section{Proof of Theorem 1}

Let $F=\frac{f^{n}}{a}, G=\frac{\left[f^{(k)}\right]^{m}}{a}$, then

$$
F-1=\frac{f^{n}-a}{a}, G-1=\frac{\left[f^{(k)}\right]^{m}-a}{a} .
$$

From the definitions of $F$ and $G$, we get

$$
\begin{aligned}
& N_{E}^{1)}\left(r, \frac{1}{F-1}\right)=N_{E}^{1)}\left(r, \frac{1}{G-1}\right)+S(r, f), N_{E}^{(2}\left(r, \frac{1}{F-1}\right) \\
&= N_{E}^{(2}\left(r, \frac{1}{G-1}\right)+S(r, f), \\
& \bar{N}_{L}\left(r, \frac{1}{F-1}\right) \leq \bar{N}\left(r, \frac{1}{F}\right)+\bar{N}(r, F)+S(r, F), \\
& \bar{N}\left(r, \frac{1}{F-1}\right)= \bar{N}\left(r, \frac{1}{G-1}\right)+S(r, F) \\
&= N_{E}^{1)}\left(r, \frac{1}{F-1}\right)+N_{E}^{(2}\left(r, \frac{1}{F-1}\right) \\
&+\bar{N}_{L}\left(r, \frac{1}{F-1}\right)+\bar{N}_{L}\left(r, \frac{1}{G-1}\right)+S(r, F) .
\end{aligned}
$$

We will distinguish two cases below.

Case $1 H \not \equiv 0$. From (2.1) it is easy to see that $m(r, H)=S(r, f)$.

Subcase 1.1. Suppose that $f^{n}$ and $\left[f^{(k)}\right]^{m}$ share $a(z)$ IM. According to (3.1), $F$ and $G$ share 1 IM except the zeros and poles of $a(z)$. By (3.1), we have

$$
\bar{N}(r, F)=\bar{N}(r, f)+S(r, f), \bar{N}(r, G)=\bar{N}(r, f)+S(r, f) .
$$

Let $z_{0}$ be a common zero of $F-1$ and $G-1$, but $a\left(z_{0}\right) \neq 0, \infty$. Through a simple calculation we know that $z_{0}$ is a zero of $H$, so

(3.6) $N_{E}^{1)}\left(r, \frac{1}{F-1}\right) \leq N\left(r, \frac{1}{H}\right)+S(r, f) \leq T(r, H)+S(r, f) \leq N(r, H)+S(r, f)$. 
From(3.4)-(3.6), lemma 2.1 and lemma 2.2, we have

$$
\begin{aligned}
\bar{N}\left(r, \frac{1}{G-1}\right) \leq & \bar{N}(r, F)+2 \bar{N}_{L}\left(r, \frac{1}{F-1}\right)+2 \bar{N}_{L}\left(r, \frac{1}{G-1}\right)+\bar{N}_{(2}\left(r, \frac{1}{F}\right) \\
& +\bar{N}_{(2}\left(r, \frac{1}{G}\right)+\bar{N}_{E}^{(2}\left(r, \frac{1}{F-1}\right)+\bar{N}_{0}\left(r, \frac{1}{F^{\prime}}\right) \\
& +\bar{N}_{0}\left(r, \frac{1}{G^{\prime}}\right)+S(r, f) \\
\leq & \bar{N}(r, f)+2 \bar{N}\left(r, \frac{1}{F^{\prime}}\right)+2 \bar{N}_{L}\left(r, \frac{1}{G-1}\right)+\bar{N}_{(2}\left(r, \frac{1}{G}\right) \\
& +\bar{N}_{0}\left(r, \frac{1}{G^{\prime}}\right)+S(r, f) .
\end{aligned}
$$

It follows by the second fundamental theorem, and (3.5), we get

$$
\begin{aligned}
T(r, G) & \leq \bar{N}(r, G)+\bar{N}\left(r, \frac{1}{G}\right)+\bar{N}\left(r, \frac{1}{G-1}\right)-N_{0}\left(r, \frac{1}{G^{\prime}}\right)+S(r, G) \\
& \leq 2 \bar{N}(r, f)+2 \bar{N}\left(r, \frac{1}{F^{\prime}}\right)+2 \bar{N}\left(r, \frac{1}{G^{\prime}}\right)+\bar{N}\left(r, \frac{1}{G}\right)+S(r, f) .
\end{aligned}
$$

By lemma 2.4, we can get

$$
T(r, G) \leq 4 \bar{N}(r, f)+2 \bar{N}\left(r, \frac{1}{\left(f^{n} / a\right)^{\prime}}\right)+2 N_{2}\left(r, \frac{1}{f^{(k)}}\right)+\bar{N}\left(r, \frac{1}{f^{(k)}}\right)+S(r, f),
$$

and by lemma 2.3 we have

$$
T\left(r, f^{(k)}\right) \leq \frac{1}{m}\left[4 \bar{N}(r, f)+2 \bar{N}\left(r, \frac{1}{\left(f^{n} / a\right)^{\prime}}\right)+2 N_{2}\left(r, \frac{1}{f^{(k)}}\right)+\bar{N}\left(r, \frac{1}{f^{(k)}}\right)\right]+S(r, f),
$$

which contradicts (1.1).

Subcase 1.2. Suppose that $f^{n}$ and $\left[f^{(k)}\right]^{m}$ share $a(z)$ CM.

Noting that $\left.N_{1)}(r, 1 / F-1)=N_{1}\right)(r, 1 / G-1)+S(r, f)$. let $z_{0}$ be a common zero of $F-1$ and $G-1$, but $a\left(z_{0}\right) \neq 0, \infty$. by a simple calculation, we can still get $H\left(z_{0}\right)=0$. Therefore

$$
N_{1)}\left(r, \frac{1}{F-1}\right) \leq N\left(r, \frac{1}{H}\right)+S(r, f) \leq N(r, H)+S(r, f) .
$$

Noting that $N_{1)}\left(r, \frac{1}{F-1}\right)=N_{1)}\left(r, \frac{1}{G-1}\right)+S(r, f)$, by (3.4) and (3.8), we can deduce

$$
\begin{aligned}
\bar{N}\left(r, \frac{1}{G-1}\right) \leq & \bar{N}(r, F)+\bar{N}_{(2}\left(r, \frac{1}{F}\right)+\bar{N}_{(2}\left(r, \frac{1}{G}\right)+\bar{N}_{0}\left(r, \frac{1}{F^{\prime}}\right) \\
& +\bar{N}_{0}\left(r, \frac{1}{G^{\prime}}\right)+\bar{N}_{(2}\left(r, \frac{1}{F-1}\right)+S(r, f) .
\end{aligned}
$$


By the second fundamental theorem, (3.5) and lemma (2.2), we have

$$
\begin{aligned}
T(r, G) & \leq \bar{N}(r, G)+\bar{N}\left(r, \frac{1}{G}\right)+\bar{N}\left(r, \frac{1}{G-1}\right)-N_{0}\left(r, \frac{1}{G^{\prime}}\right)+S(r, G) \\
& \leq 2 \bar{N}(r, f)+N_{2}\left(r, \frac{1}{G}\right)+\bar{N}\left(r, \frac{1}{F^{\prime}}\right)+S(r, f) .
\end{aligned}
$$

Then by lemma 2.4 we have

$$
m T\left(r, f^{(k)}\right) \leq 2 \bar{N}(r, f)+\bar{N}\left(r, 1 /\left(f^{n} / a\right)^{\prime}\right)+N_{2}\left(r, \frac{1}{f^{(k)}}\right)+S(r, f) .
$$

This contradicts (1.2).

Case 2. $H \equiv 0$. Integration yields

$$
\frac{1}{F-1} \equiv \frac{A}{G-1}+B .
$$

where $A, B$ are constants and $A \neq 0$. It is easy to see that $F$ and $G$ share $1 \mathrm{CM}$. Now we claim $B=0$.

If $\bar{N}(r, f) \neq S(r, f)$, then by (3.10), we get $B=0$. So our claim holds. Hence we can assume

$$
\bar{N}(r, f)=S(r, f)
$$

since $B \neq 0$, then we can rewrite $(3.10)$ as

$$
\frac{1}{F-1} \equiv \frac{B(G-1+A / B)}{G-1}
$$

So

$$
\bar{N}\left(r, \frac{1}{G-1+A / B}\right)=\bar{N}(r, F)=S(r, f) .
$$

If $A \neq B$, by the second fundamental theorem and (3.12) we have

$$
\begin{aligned}
T(r, G) & \leq \bar{N}(r, G)+\bar{N}\left(r, \frac{1}{G}\right)+\bar{N}\left(r, \frac{1}{G-1+A / B}\right)+S(r, f) \\
& \leq \bar{N}\left(r, \frac{1}{G}\right)+S(r, f) \leq T(r, G)+S(r, f) .
\end{aligned}
$$

Hence

$$
\begin{aligned}
& T(r, G)=\bar{N}\left(r, \frac{1}{G}\right)+S(r, f), \quad \text { i.e. }, \\
& m T\left(r, f^{(k)}\right)=\bar{N}\left(r, \frac{1}{f^{(k)}}\right)+S(r, f) .
\end{aligned}
$$


This is a contradiction with (1.1) and (1.2). If $A=B$, then from (3.1) we get $\frac{1}{F-1}=\frac{A G}{G-1}$. Rewrite it as

$$
-\frac{a^{2}}{f^{m}\left(A f^{n}-a-a A\right)} \equiv \frac{\left[f^{(k)}\right]^{m}}{f^{m}} .
$$

So by lemma 2.4 , we have

$$
\begin{aligned}
(m+n+1) T(r, f) & =T\left(r,\left(\frac{f^{(k)}}{f}\right)^{m}\right)+S(r, f) \\
& \leq m\left[N\left(r, \frac{1}{f}\right)+k \bar{N}(r, f)\right]+S(r, f) \leq m T(r, f)+S(r, f) .
\end{aligned}
$$

This implies $T(r, f)=S(r, f)$ since $n, m \geq 1$. which is a contradiction. Hence our claim is right. So $(G-1) /(F-1)=A$. Theorem 1 is thus completely proved.

\section{Proof of Theorem 2}

The proof of Theorem 2 is partly similar to the proof of Theorem 1, Let $F$ and $G$ defined as Theorem 1, hence we have (3.1)-(3.5). We still distinguish two cases.

Case $1 H \not \equiv 0$.

Subcase 1.1. Suppose that $f^{n}$ and $\left[f^{(k)}\right]^{m}$ share $a(z)$ IM, then we can still get (3.6), and by (3.4)-(3.6) and lemma 2.2 we have (3.7).

then by the second fundamental theorem, (3.5) and (3.7), we have

$$
\begin{aligned}
T(r, F) & \leq \bar{N}(r, F)+\bar{N}\left(r, \frac{1}{F}\right)+\bar{N}\left(r, \frac{1}{F-1}\right)-N_{0}\left(r, \frac{1}{F^{\prime}}\right)+S(r, F) \\
& \leq 2 \bar{N}(r, f)+2 \bar{N}\left(r, \frac{1}{G^{\prime}}\right)+2 \bar{N}\left(r, \frac{1}{F^{\prime}}\right)+\bar{N}\left(r, \frac{1}{F}\right)+S(r, f) .
\end{aligned}
$$

Applying lemma 2.3 and lemma 2.4 to the above inequality, we get

$$
n T(r, f) \leq(2 k+6) \bar{N}(r, f)+3 \bar{N}\left(r, \frac{1}{f}\right)+2 N_{k+2} N\left(r, \frac{1}{f}\right)+S(r, f) .
$$

This implies

$$
(2 k+6) \Theta(\infty, f)+3 \Theta(0, f)+2 \delta_{k+2}(0, f) \leq 2 k+11-n .
$$

This contradicts (1.3)

Subcase 1.2. Suppose that $f^{n}$ and $\left[f^{(k)}\right]^{m}$ share $a(z)$ CM. From the above discuss, we can easily obtain $N_{1)}\left(r, \frac{1}{F-1}\right)=N_{1)}\left(r, \frac{1}{G-1}\right)+S(r, f)$, by lemma 2.1, we can deduce

$$
\begin{aligned}
\bar{N}\left(r, \frac{1}{F-1}\right) \leq & \bar{N}(r, F)+\bar{N}_{(2}\left(r, \frac{1}{F}\right)+\bar{N}_{(2}\left(r, \frac{1}{G}\right)+\bar{N}_{0}\left(r, \frac{1}{F^{\prime}}\right) \\
& +\bar{N}_{0}\left(r, \frac{1}{G^{\prime}}\right)+\bar{N}_{(2}\left(r, \frac{1}{G-1}\right)+S(r, f) .
\end{aligned}
$$


So use the second fundamental theorem again and we get

$$
n T(r, f) \leq\left[(k+5)-(k+3) \Theta(\infty, f)+\delta_{2}(0, f)+\delta_{k+2}(0, f)\right] T(r, f)+S(r, f) .
$$

This is a contradiction.

Case 2. $H \equiv 0$

Similarly, we can also get (3.10). Next we claim $B=0$. If $\bar{N}(r, f) \neq S(r, f)$, then it follows that $B=0$ from (3.10). Hence, we may assume that (3.11) holds. If $B \neq 0, B \neq-1$. then

$$
\frac{A}{G-1} \equiv-\frac{B F-(B+1)}{F-1},
$$

and so

$$
N(r, G)=\bar{N}\left(r, \frac{1}{F-(B+1) / B}\right) .
$$

Again by second fundamental theorem and (4.5) we have

$$
\begin{gathered}
T(r, F)=\bar{N}\left(r, \frac{1}{F}\right)+S(r, f) . \quad \text { i.e. } \\
n T(r, f) \leq \bar{N}\left(r, \frac{1}{F}\right)+S(r, f) \leq T(r, f)+S(r, f) .
\end{gathered}
$$

If $n \geq 2$, then we have $T(r, f)=S(r, f)$. This is a contradiction. If $n=1$, then we have $\bar{N}\left(r, \frac{1}{f}\right)=T(r, f)+S(r, f)$, and it follows that $\Theta(0, f)=0$ and from (3.11), (1.3), (1.4) we may deduce $\delta_{k+2}(0, f)>1$, it is impossible. So we can assume $B=-1$, then we can get

$$
\frac{\left[f^{(k)}\right]^{m}}{a}-(A+1) \equiv-A \cdot a \cdot \frac{1}{f^{n}} .
$$

Therefore, by(4.5), we get

$$
n T(r, f)=T\left(r,\left[f^{(k)}\right]^{m}\right)+S(r, f) \leq m T(r, f)+S(r, f) .
$$

It shows that $n \leq m$.

If $A=-1$, by (4.3), we have $f \cdot\left[f^{(k)}\right]^{m} \equiv a^{2}$, which with the above inequality may lead to $n=m=1$, from Theorem E. We can get our claim. If $A \neq-1$. By second fundamental theorem, lemma 2.3, (3.11) and (4.3) we have

$$
\begin{aligned}
T\left(r,\left[f^{(k)}\right]^{m}\right) & \leq \bar{N}\left(r, \frac{1}{\left[f^{(k)}\right]^{m}-a(A+1)}\right)+\bar{N}\left(r, \frac{1}{f^{(k)}}\right)+S(r, f) \\
& \leq k \bar{N}(r, f)+N_{k+2}\left(r, \frac{1}{f}\right)+S(r, f) \leq T(r, f)+S(r, f) .
\end{aligned}
$$

which with (4.4) may deduce $m=1$, thus from Theorem E, we can get our claim. Hence our claim $B=0$ holds. 
Next we will proof $A=1$. From (3.12) we have $G-1 \equiv A(F-1)$ then

$$
\bar{N}\left(r, \frac{1}{G}\right)=\bar{N}\left(r, \frac{1}{F+1 / A-1}\right) .
$$

If $A \neq 1$, by second fundamental theorem, we have

It implies

$$
\begin{gathered}
T(r, F) \leq \bar{N}(r, F)+\bar{N}\left(r, \frac{1}{F}\right)+\bar{N}\left(r, \frac{1}{G}\right)+S(r, f) . \quad \text { i.e. }, \\
n T(r, f) \leq \bar{N}(r, f)+\bar{N}\left(r, \frac{1}{f}\right)+N_{k+2}\left(r, \frac{1}{f}\right)+S(r, f) .
\end{gathered}
$$

$$
\Theta(\infty, f)+\Theta(0, f)+\delta_{k+2}(0, f) \leq 3-n
$$

Combining (4.5) with (1.3) yields $\Theta(0, f)>1$, since $n \geq 1$. This is a contradiction. Hence $A=1$ and $f^{n} \equiv\left[f^{(k)}\right]^{m}$. Now Theorem 2 has been completely proved.

\section{References}

[1] W. K. Hayman, Meromorphic Functions, Clarendon Press, Oxford, 1964.

[2] L. Yang, Value Distribution Theory, Springer-Verlag, Berlin, 1993.

[3] C. C. Yang and H.X. Yi, Uniqueness Theory of Meromorphic Functions, Science Press, Beijing; Kluwer Academic Publishers, New York, 2003.

[4] G. G. Gundersen and L. Z. Yang, Entire functions that share one value with one or two of their derivatives, J. Math. Anal. Appl., 223 (1998), 88C95.

[5] I. Lahiri and A. Sarkar, Uniqueness of a meromorphic function and its derivative, J. Inequal. Pure Appl. Math., 5(1) (2004), p. 9, Art. 20.

[6] R. Brück, On entire functions which share one value with their first derivatives, Result in Math., 30 (1996), pp. 21-24.

[7] L. Z. Yang, Solution of a differential equation and its applications, Kodai Math. J., 22 (1999), pp. 458-464.

[8] Q. C. Zhang, The uniqueness of meromorphic functions with their derivatives, Kodai Math. J., 21 (1998), pp. 179-184.

[9] K. W. Yu, On entire and meromorphic functions that share small functions with their derivatives, J. Inequal. Pure Appl. Math.,4(1) (2003), p. 7, Art. 21.

[10] T. D. Zhang and W. R. Lü, Notes on a meromorphic function sharing one small function with its derivative,Complex Variables and Elliptic Equations Vol. 53, No. 9, (2008), 857-867

[11] C. C. Yang, On deficiencies of differential polynomials II, Math. Z., 125 (1972), pp. 107-112. 